\title{
On the Microscopic Flow Characteristics of Nanofluids by Molecular Dynamics Simulation on Couette Flow
}

\author{
Wenzheng Cui ${ }^{*}$, , Minli Bai ${ }^{1}, \mathrm{Jizu}_{\mathrm{Lv}}{ }^{1}$ and Xiaojie $\mathrm{Li}^{2}$ \\ ${ }^{1}$ School of Energy and Power Engineering, Dalian University of Technology, Dalian 116024, China \\ ${ }^{2}$ The State Key Laboratory of Structural Analysis for Industrial Equipment, Dalian University of Technology, Dalian \\ 116024, China
}

\begin{abstract}
Adding a small amount of nanoparticles to conventional fluids (nanofluids) has been proved to be an effective way for improving capability of heat transferring in base fluids. The change in micro structure of base fluids and micro motion of nanoparticles may be key factors for heat transfer enhancement of nanofluids. Therefore, it is essential to examine these mechanisms on microscopic level. The present work performed a Molecular Dynamics simulation on Couette flow of nanofluids and investigated the microscopic flow characteristics through visual observation and statistic analysis. It was found that the even-distributed liquid argon atoms near solid surfaces of nanoparticles could be seemed as a reform to base liquid and had contributed to heat transfer enhancement. In the process of Couette flow, nanoparticles moved quickly in the shear direction accompanying with motions of rotation and vibration in the other two directions. When the shearing velocity was increased, the motions of nanoparticles were strengthened significantly. The motions of nanoparticles could disturb the continuity of fluid and strengthen partial flowing around nanoparticles, and further enhanced heat transferring in nanofluids.
\end{abstract}

Keywords: Nanofluids, molecular dynamics method, couette flow, mechanism, heat transfer enhancement.

\section{INTRODUCTION}

Nanofluids have received much attention in recent years due to the significantly improved heat transferring ability compared to conventional fluids, which offer important economic benefits. The concept of nanofluids refers to the stable suspensions engineered by suspending nanoparticles of metal, metallic oxide or non-metallic with average sizes below $100 \mathrm{~nm}$ in traditional heat transfer fluids, which was first introduced by Choi [1] from Argonne National Laboratory in 1995. In particular, a spot of uniformly dispersed and stably suspended nanoparticles could dramatically improve the thermal properties of base fluids. This new class of nanotechnology-based heat transfer fluids could meet the requirements of ultrahigh-performance cooling in many industrial technologies. The increasing interest in nanofluids has heightened the need for bringing out comprehensively the fluid dynamics and heat transfer theories of nanofluids. Of particular interest and complexity are the mechanisms of heat transfer enhancement in flowing nanofluids. Theories of fluid dynamics and heat transfer in macroscale are based on continuous medium hypothesis. They are not suitable for nanofluids, for when it comes to nanoscale, small size effect appears and surface forces tend to predominate in fluids. Furthermore, the continuity of fluid flowing damaged by nanoparticles in nanofluids and intermolecular impact effects should also be considered. However, much research in recent years has focused on

*Address correspondence to this author at the School of Energy and Power Engineering, Dalian University of Technology, Dalian 116024, China; Tel: +86-411-84706305, +86-13842695965; Fax: +86-411-84706305; E-mail: cuiwenzheng@mail.dlut.edu.cn thermal conductivity of nanofluids. Although it's important, it is even more important to probe into the flow characteristics of nanofluids since they are designed as substitute for conventional cooling fluids.

Molecular dynamics (MD) method is an effective way for examining the microscopic mechanisms of flow characteristics and heat transfer enhancement in flowing nanofluids. The basic principle of MD method is solving molecular (or atomic) Newton equations of motion regarding effects of interaction potential between molecules (or atoms) and external restriction. By this method, time-evolving microscopic process of system is simulated; and equilibrium parameters and transport properties could be statistical computed. Previous researchers have utilized MD method to study the mechanisms of heat transfer and fluid flow from the microcosmic aspect and made a few achievements. Vergeles et al. [2, 3] studied the motor behavior of fluid containing nanoparticles in semi-infinite space and kinetic behavior when particles approaching the wall boundary by MD method. And the study demonstrated MD method was effective to describe the motions of nanoparticles. G. Nagayama and P. Cheng [4] studied the effect of interface wettability on the pressure driven flow of LJ fluid in nanochannel by MD method and the results showed the hydrodynamic boundary condition at the solid-liquid interface depended on both the interface wettability and the magnitude of the driving force. Xue et al. [5] studied how the ordering of the liquid at the liquid-solid interface affected the interfacial thermal resistance using non-equilibrium MD simulations in which a temperature gradient was imposed. Reza Kamali and Ali Kharazmi [6] developed a molecular based scheme for simulating of surface roughness effects on nano- and micro- scale flows. Ahadian et al. [7] performed 
MD simulations to evaluate the penetration of two different fluids through a designed nanochannel. It was revealed that the wall-fluid interaction plays a significant role in such transport phenomena, namely, fluid flow in nanochannels. S. C. Maroo and J. N. Chung [8] numerically simulated heating/cooling of fluid atoms by wall atoms and compared MD simulation results to the analytical solution of 1-D heat equation. The results were found to match well with the analytical solution.

Plenty of literatures based on MD simulation of liquids have been reported; however, few of them focus on the simulation of flow characteristics in nanofluids flow. Since these are important for understanding the mechanism of enhanced heat transferring in nanofluids, based on our previous studies the present paper presents a MD simulation on Couette flow of $\mathrm{Cu}$-Ar nanofluids and discusses in detail the microscopic flow characteristics of nanofluids. A MD simulation program adapted from the classic MD simulator named Lammps [9] is performed for the simulation. The authors attempt to examine the flow characteristics and mechanisms of heat transfer enhancement in nanofluids.

\section{SIMULATION MODEL AND METHOD}

MD method is a deterministic simulation method for studying physical phenomena. With a given potential MD simulation solves Newton's equation of motion for a system of particles and gives accurate calculation results for the movement of particles at atomic level. However, although the result of MD method is accurate and reliable it can only afford simulation for a region of very small size, which is normally in nanoscale. The reason is mainly because the solving of Newton's equation of motion for a large system is usually a time-consuming job. Therefore, researchers that using MD method to carry out studies usually established simulation model of relatively small size [4-8]. Besides, the aim of the present work is to study microscopic flow characteristics of nanofluids and examine micro motions of nanoparticles in the nanofluids, therefore considering a simulation region with a relatively small size is enough for the current study. The present simulation model consists of two parallel plates and nanofluid between them, as shown in Fig. (1). The geometric model of simulation box has the size of $5.74 \times 24.1 \times 28.4 \mathrm{~nm}^{3}$ and the distance between two plates is $27 \mathrm{~nm}$. The present size of model has been considered to be effective based on our preliminary study. The nanofluid is comprised of argon atoms as base fluid and four rows of spherical copper nanoparticles with diameter of $4 \mathrm{~nm}$. Liquid argon is chosen to be base liquid for its stability and simple potential function which avoids dealing with unnecessary complex interactions. All the atoms in simulation box is in the face-centered cubic (FCC) lattice initially and the total amount of atoms in the model is 120600 . NTV ensemble has been chosen for simulation and the temperature of simulation system is fixed to be $86 \mathrm{~K}$. When the simulation of Couette flow begins, both parallel plates are given the same translational velocity but in opposite directions along the yaxis, as shown in Fig. (1). And the model has been simulated twice with shearing velocities of $10 \mathrm{~m} / \mathrm{s}$ and $50 \mathrm{~m} / \mathrm{s}$ respectively, for comparing the flowing behaviors of nanofluids.

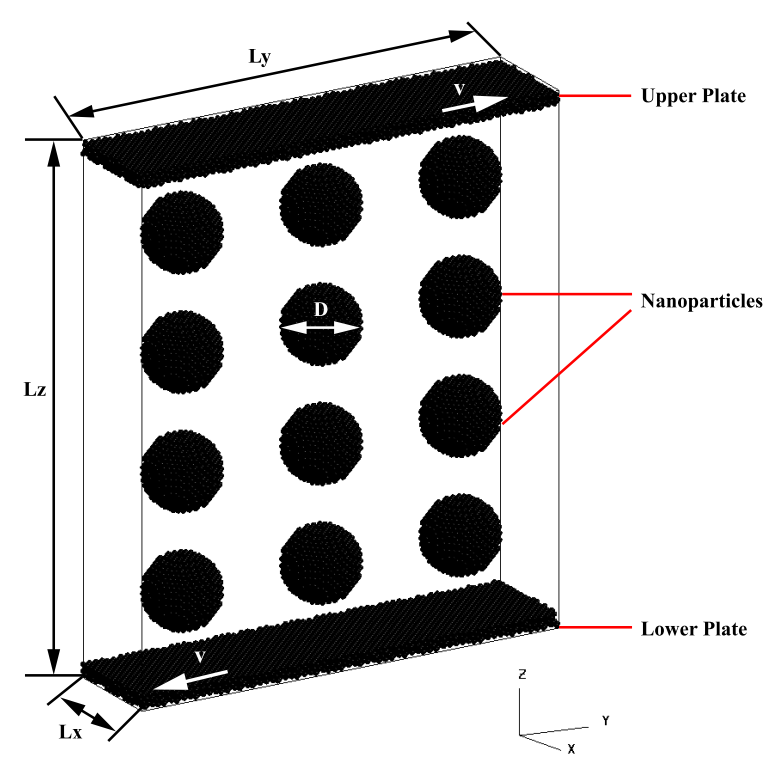

Fig. (1). Schematic diagram of simulation model.

Suitable potential functions are important factors to ensure the MD simulation results be accurate and reliable. Presently empirical or semi-empirical correlations are adopted in most classic MD simulations. Among them, Lennard-Jones (LJ) potential function is the most commonly known one which is frequently used to describe the interactions between atoms or molecules of liquids and gases. $\mathrm{LJ}$ potential function $\left(\mathrm{u}_{\mathrm{ij}}\right)$ is given by

$u_{i j}=4 \varepsilon\left[\left(\frac{\sigma}{r_{i j}}\right)^{12}-\left(\frac{\sigma}{r_{i j}}\right)^{6}\right]$

where $r_{i j}$ represents the intermolecular distance between atoms $i$ and $j\left(r_{i j}=r_{j}-r_{i}\right) . \varepsilon$ and $\sigma$ are parameters describing the bonding energy and bonding distance respectively, and both of them depend on the type of molecules. For argon, the LJ parameters are $\varepsilon=1.67 \times 10^{-21} \mathrm{~J}$ and $\sigma=0.3405 \mathrm{~nm}$ [10]. For copper, the LJ parameters are $\varepsilon=65.625 \times 10^{-2} \mathrm{~J}$ and $\sigma=0.2338 \mathrm{~nm}[10]$. The first term in the above equation on the right side represents the strong repulsion caused by inner electrons or ion overlap and the second one represents electrostatic interaction between dipoles. Parameters between argon atoms and copper atoms are calculated according to Lorentz-Berthlot mixing law [11] which is given by

$$
\begin{aligned}
& \sigma_{C u-A r}=\left(\sigma_{C u}+\sigma_{A r}\right) / 2 \\
& \varepsilon_{C u-A r}=\sqrt{\varepsilon_{C u} \varepsilon_{A r}}
\end{aligned}
$$

Therefore, $\varepsilon$ and $\sigma$ between copper and argon atoms are $10.4153 \times 10^{-21} \mathrm{~J}$ and $0.2872 \mathrm{~nm}$, respectively.

The MD simulation is performed on a four core parallel computer. The cut-off ratio is chosen to be $2.5 \sigma_{\mathrm{Ar}}$ and periodic boundary conditions are applied along the $\mathrm{x}-$ and $\mathrm{y}-$ directions. The atoms in simulation box has an initial manmade distribution, therefore it needs to be relaxed adequately which allows the system to adapt itself to a more natural balance condition. In the present simulation, it is relaxed for 
600 ps with each time step length of 2 fs. The computer running time of relaxation takes about 48 hours and the energy distribution in relaxation process is shown in Fig. (2). The enthalpy of system trends to converge which indicates the system reaches the equilibrium state. The MD simulation model after relaxation in which the atoms are in the relative equilibrium position is shown in Fig. (3). In the figure, nanoparticles at different positions in flow field are labeled $\mathrm{A}$ and $\mathrm{B}$ for the following discussions. After the relaxation, both plates are given the same translational velocity in opposite directions along the y-axis. And the model is simulated twice with different shearing velocities of $10 \mathrm{~m} / \mathrm{s}$ and $50 \mathrm{~m} / \mathrm{s}$ respectively. The simulation time of Couette flow is $1600 \mathrm{ps}$, with the same length of time step as relaxation, and the computer running time takes about 130 hours.

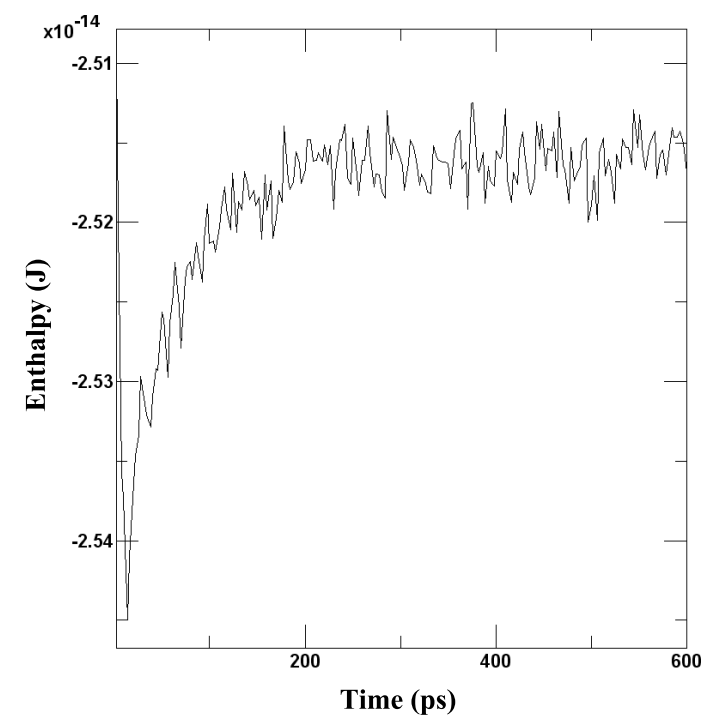

Fig. (2). Energy distribution in relaxation process.

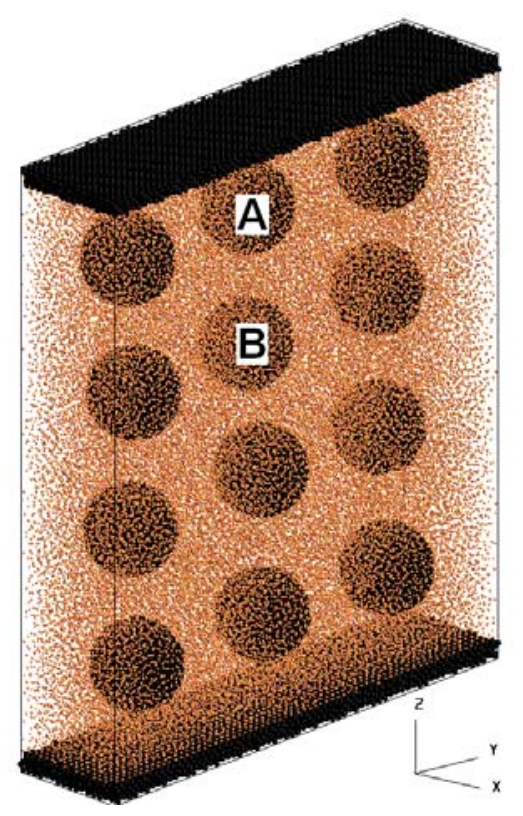

Fig. (3). The whole MD simulation model.

\section{RESULTS AND DISCUSSIONS}

\subsection{Absorption Layer}

In the process of MD simulation, the strong interactive force between copper atoms holds them together and the atoms maintain the shape of nanoparticles during the shearing flow simulation. Similarly, the interactive force between copper and argon atoms is much stronger than that between argon atoms, therefore argon atoms surrounding a nanoparticle is absorbed to it and move accompanying the nanoparticle, and in Fig. (4) the absorption layer is schematically illustrated. Influenced by the even distribution of solid atoms, distribution of argon atoms in the absorption layer is more uniform compared to the free atoms in base fluid. The observed phenomenon of molecular layering at the liquid-solid interface agrees with the study of Li et al. [12]. And by the number density method they proposed which is defined as the number of atoms per unit volume, the density distribution of liquid argon from the surface of plates is examined. The number density is given by

$n=\frac{\Delta N}{\Delta V}$

where $\Delta \mathrm{N}$ is the number of atoms within the volume $\Delta \mathrm{V}$. The computational domain considered a distance of $2 \mathrm{~nm}$ from the plate along $\mathrm{z}$ - axis. The authors repeatedly used region and group commands in Lammps software to divide equably 9 cubic thin regions from $0.2 \mathrm{~nm}$ to a distance of 2.0 $\mathrm{nm}$, therefore each thin region is $0.2 \mathrm{~nm}$ thickness. At specific simulation time steps, the number of argon atoms in each thin region is counted and outputted. Therefore according to equation (4) one gets the number density distribution of liquid argon near plates. The authors present the result as a ratio of number density distributions of absorbed argon atoms and free argon atoms.

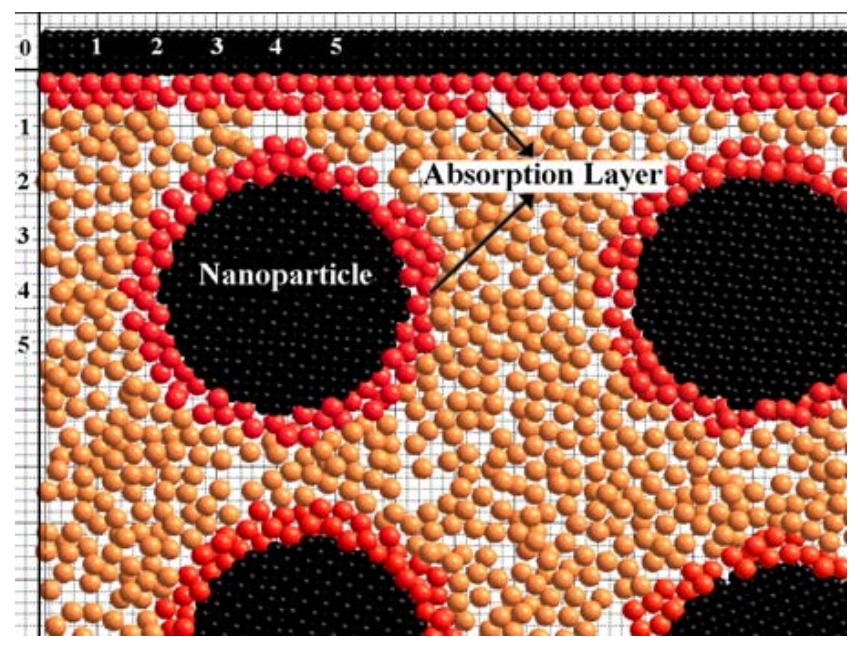

Fig. (4). Absorption Layer near surfaces of nanoparticles and plates.

In Fig. (5) the ratios of number density distribution against the distance from the solid surface of plate ( $d$ in the figure) at simulation time of $1000 \mathrm{ps}, 1100 \mathrm{ps}$, and $1200 \mathrm{ps}$ are presented. Through analysis of the density distribution it is found the density linearly decreases with the decreasing of distance from solid surface. At the distance of about $1 \mathrm{~nm}$ 
the density is close to that of liquid argon which indicates the average thickness of absorption layer is about 0.4-0.6 nm.

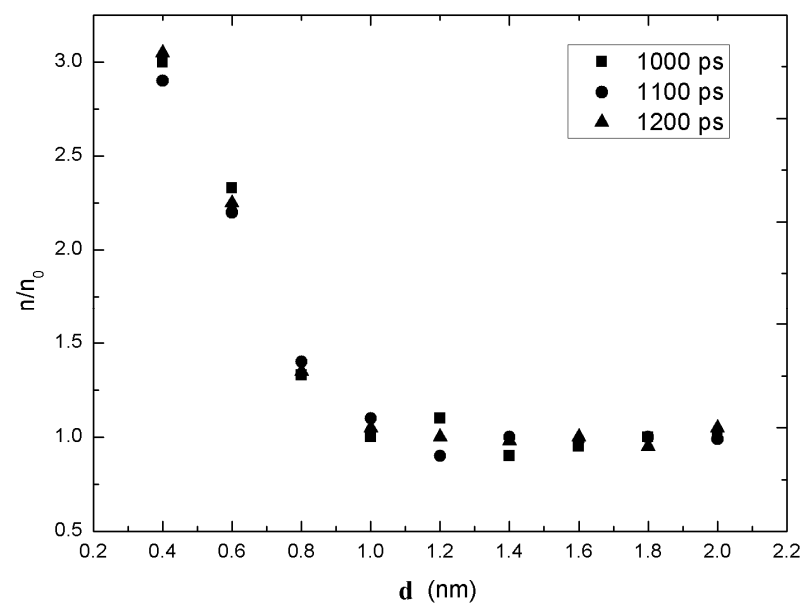

Fig. (5). Density distribution of argon.

The even-distributed absorption layer is believed to have a much higher thermal conductivity than that of liquid which can be seemed as a reform for base liquid, and will be effective for heat transferring between liquid and solid. However, the effective diameter of nanoparticle is increased at the meantime for the existence of absorption layer and the nanoparticles become heavier which slows the motion of nanoparticles.

\subsection{Motions of Nanoparticles}

The motions of nanoparticles are examined by statistically computing of translational velocity and angular velocity of nanoparticles $A$ and $B$, and tracing trajectories of all the nanoparticles. In Figs. (6-9), the translational velocity components of nanoparticles $\mathrm{A}$ and $\mathrm{B}$ along three axes at different simulation time are illustrated. It could be found that along the y-axis (shearing direction) the translational velocity component of nanoparticles at different positions in the flow field is significantly larger than those along the other directions. The translational velocity of nanoparticle A along the $y$-axis is closer to the shearing velocity of plates, when the shearing velocity is $10 \mathrm{~m} / \mathrm{s}$, the average translational velocity without maximum and minimum values is $8.28 \mathrm{~m} / \mathrm{s}$; when the shearing velocity is $50 \mathrm{~m} / \mathrm{s}$, the value is $40.24 \mathrm{~m} / \mathrm{s}$. The translational velocity of nanoparticle $\mathrm{B}$ is significantly lower than A, when the shearing velocity is $10 \mathrm{~m} / \mathrm{s}$, the average translational velocity is $4.17 \mathrm{~m} / \mathrm{s}$; and when the shearing velocity is $50 \mathrm{~m} / \mathrm{s}$, the average value is $19.61 \mathrm{~m} / \mathrm{s}$. The biggest characteristic of translational velocity components along $\mathrm{x}$ - and $\mathrm{z}$-axis is the velocity components have both positive and negative values, which indicates the moving of nanoparticles along the other two directions is irregular vibration. Through analysis of translational velocity components, it could be found that for nanoparticles in the flow field at different positions the value of translational velocity component along shearing direction has a huge difference, and along the other two directions nanoparticles have irregular vibration motions. All these flow characteristics of nanoparticles make the movement speed in channel be more evenly distributed which is good for energy exchange between nanofluids and plates.

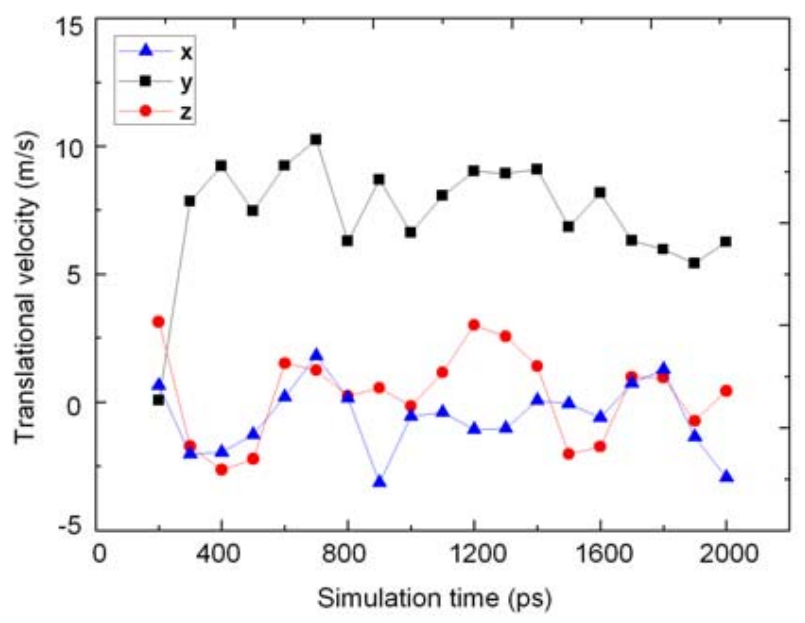

Fig. (6). Translational velocity of nanoparticle A with shearing velocity of $10 \mathrm{~m} / \mathrm{s}$.

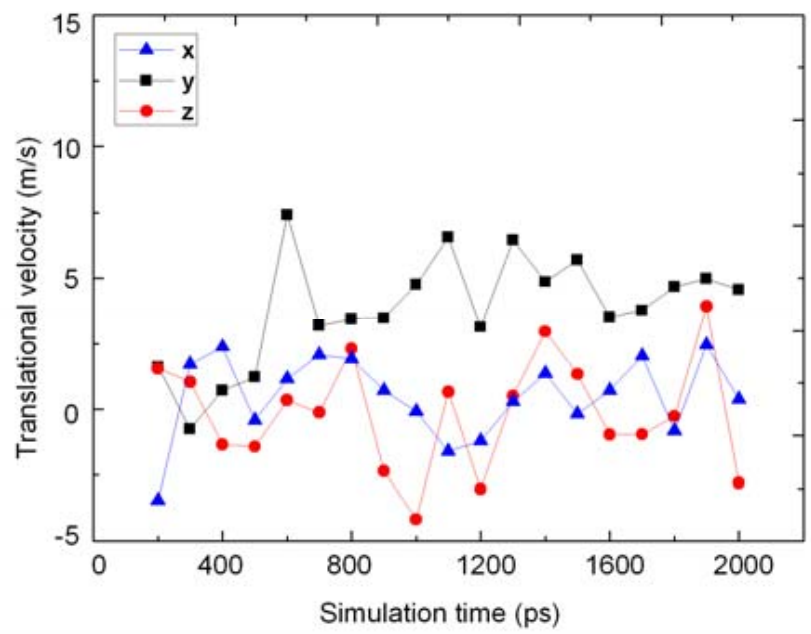

Fig. (7). Translational velocity of nanoparticle $B$ with shearing velocity of $10 \mathrm{~m} / \mathrm{s}$.

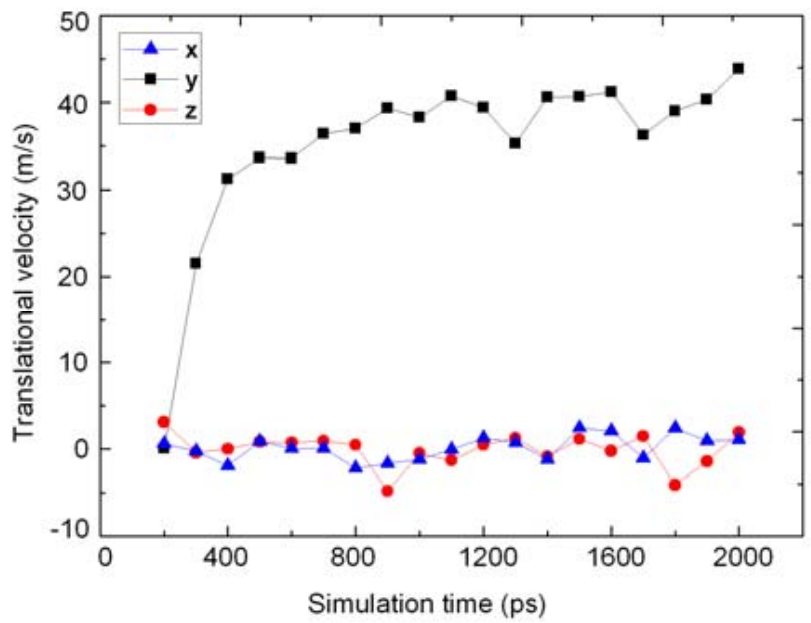

Fig. (8). Translational velocity of nanoparticle A with shearing velocity of $50 \mathrm{~m} / \mathrm{s}$.

The angular velocity components of nanoparticles at different simulation time are shown in Figs. (10-13). 
Through analysis of the statistical calculation, it can be found the variation of angular velocity of nanoparticle $B$ is more smooth which indicates that nanoparticle B is under a steady state of rotating during the flowing. By contrast, the angular velocity of nanoparticle $\mathrm{A}$ is in a state of flux which indicates the attraction from plate has great inhibition effect for it. This also further demonstrates that during the Couette flow of nanofluids, the rotation of nanoparticles is steadier in the main flow field. When the shearing velocity is increased the angular velocity of nanoparticle has also been increased. The angular velocities of nanoparticles A and B have both positive and negative values during the simulation which indicates the rotation of nanoparticles is irregular. When nanofluids flow, the rotation of nanoparticles causes slight change in surrounding fluid, and further influences the fluid flow of the whole flow field. For main flow region, this will promote the turbulent extent; and for boundary layer of a flat plate, the velocity distribution will be disturbed which further enhances energy exchange between plates and nanofluids.

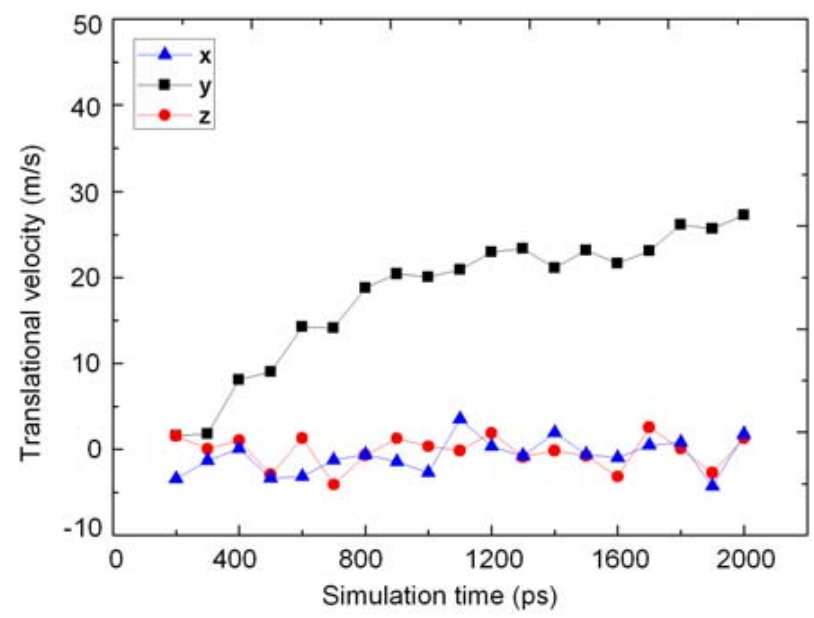

Fig. (9). Translational velocity of nanoparticle $\mathrm{B}$ with shearing velocity of $50 \mathrm{~m} / \mathrm{s}$.

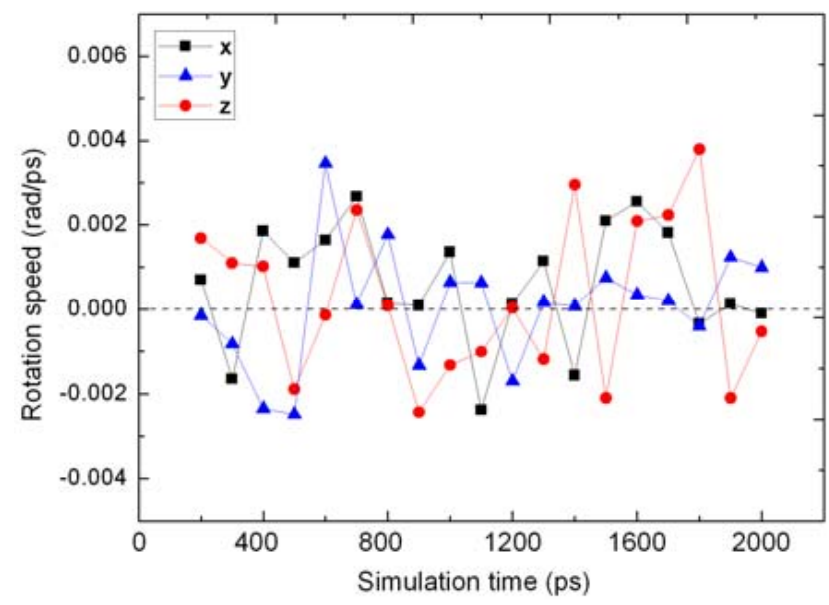

Fig. (10). Angular velocity of nanoparticle A with shearing velocity of $10 \mathrm{~m} / \mathrm{s}$.

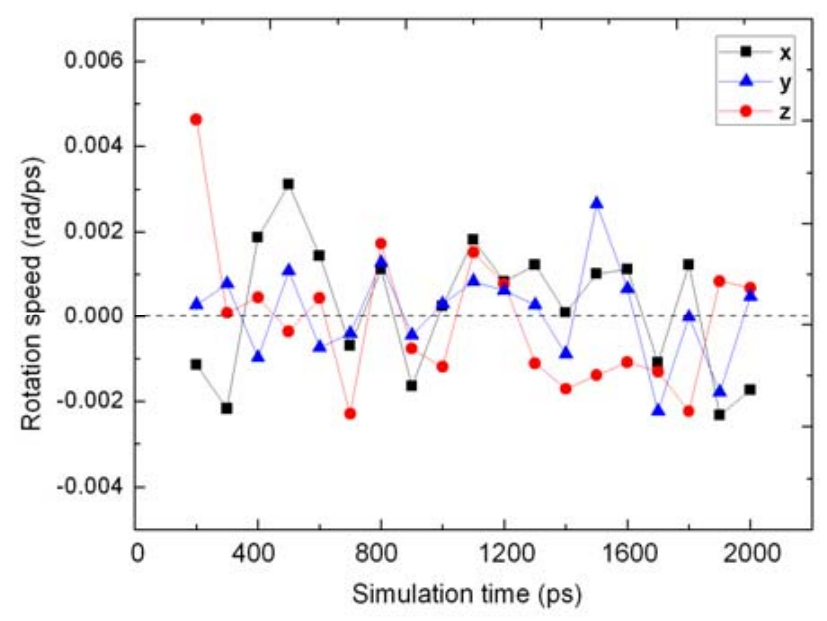

Fig. (11). Angular velocity of nanoparticle B with shearing velocity of $10 \mathrm{~m} / \mathrm{s}$.

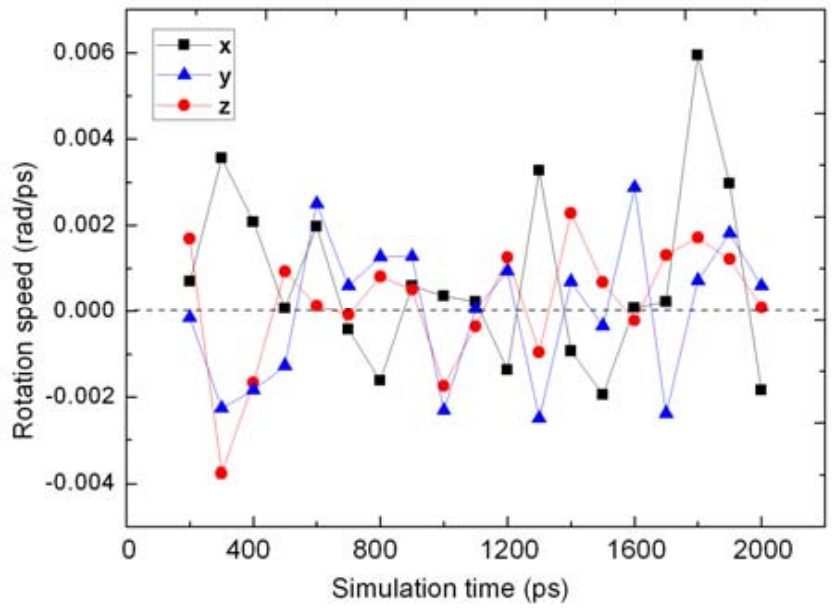

Fig. (12). Angular velocity of nanoparticle A with shearing velocity of $50 \mathrm{~m} / \mathrm{s}$.

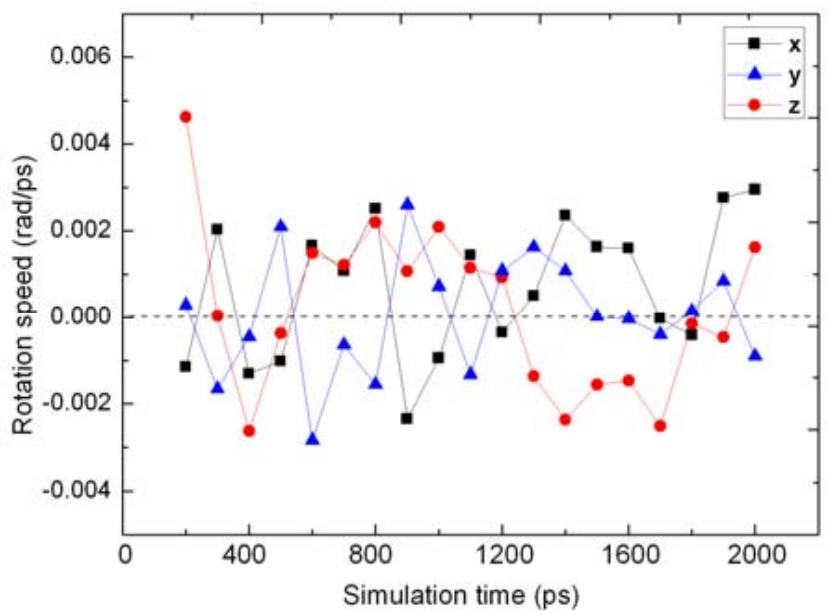

Fig. (13). Angular velocity of nanoparticle B with shearing velocity of $50 \mathrm{~m} / \mathrm{s}$. 
The trajectories of nanoparticles from 1500 ps to $1600 \mathrm{ps}$ under different shearing velocity are shown in Figs. $(\mathbf{1 4}, \mathbf{1 5})$. In the figures, nanoparticles in black color represent the original position at $1500 \mathrm{ps}$ and red color shows the trajectories. The figures further illustrate the irregular vibration and rotation of nanoparticles in $\mathrm{x}$ - and $\mathrm{z}$-axis directions besides translation driven by shearing force. For the great inhibition effect from plate, the irregular motions of nanoparticles near a plate is weaker than those ones far from the plate, which is presented in the trajectories that the displacements of nanoparticles in the main flow region is obviously greater that those close to plates. By comparison of the figures, it is found that when the shearing velocity is increased, the irregular motion extent of nanoparticles is increased. The trajectories of nanoparticles further indicate the irregular motions of nanoparticles are more obvious in the main flow field. The micro motions including rotation and vibration enhance relative motion between nanoparticles and fluid which will cause regional convection in fluid and strengthen flow and heat transfer properties of nanofluids.
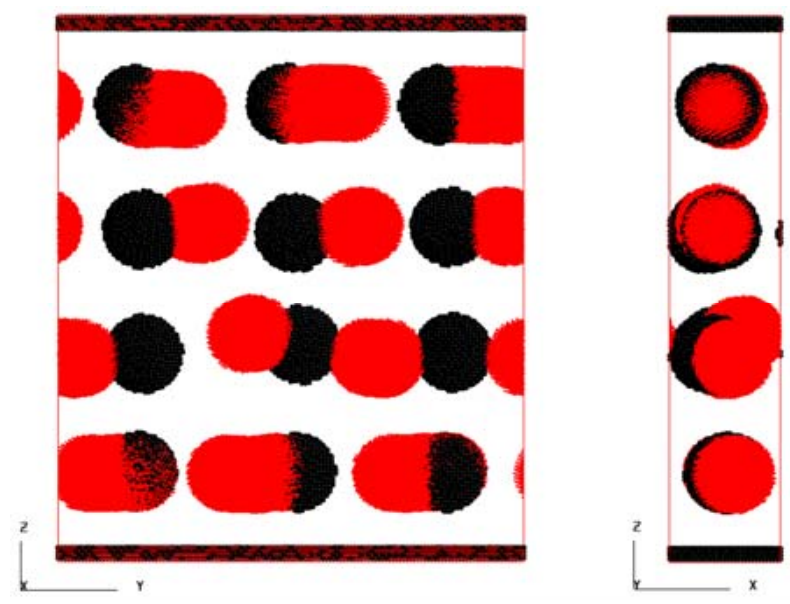

Fig. (14). Trajectories of nanoparticles from 1500 ps to 1600 ps with shearing velocity of $10 \mathrm{~m} / \mathrm{s}$.
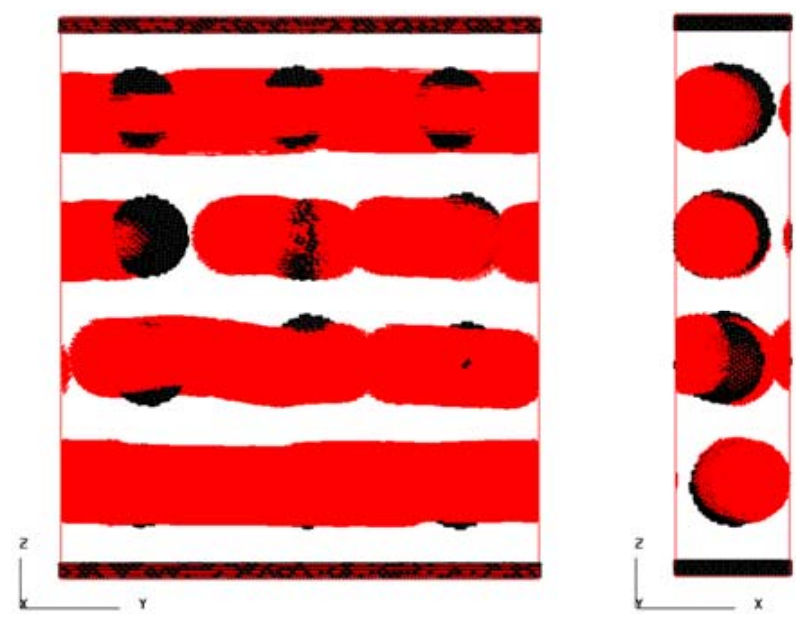

Fig. (15). Trajectories of nanoparticles from 1500 ps to 1600 ps with shearing velocity of $50 \mathrm{~m} / \mathrm{s}$.

\section{CONCLUSIONS}

In this paper, we preformed a MD simulation on micro Couette flow of nanofluids, studied the flow characteristics of nanofluids, and proposed mechanisms of heat transfer enhancement in nanofluids. Through visual observation and statistic analysis of the simulation results, the following conclusions were drawn:

1) The absorption layer comprised of even-distributed liquid argon atoms near surface of nanoparticles has a much higher thermal conductivity than that of liquid, which can be seemed as a reform for base liquid and will be effective for heat transferring between fluid and solid.

2) By statistical analysis of the motions of nanoparticles, it was found that along the shearing direction the nanoparticles at different positions have very different values of translational velocity component, and along the other two directions nanoparticles have irregular vibration motion. In the meantime, nanoparticles rotate with a certain angular velocity around three axes. The extent of vibration and rotation motions of nanoparticles increases with the increased shearing velocity of plates. Besides, motions of nanoparticles in the main flow region are more obvious which is mainly because those nanoparticles suffer less influence from the plates. The motions of nanoparticles could disturb the continuity of fluid and strengthen partial flowing around nanoparticles, and further enhance heat transferring in nanofluids.

3) The current work is a basic research for microscopic flow characteristics of nanofluids by MD method. We proposed the absorption phenomena of liquid atoms and motions of nanoparticles as main mechanisms that may be effective to enhance heat transport in nanofluids. However, whether the results and conclusions could be extended to a macroscopic scale still needs further research.

\section{ACKNOWLEDGMENTS}

The supports of the National Natural Science Foundation of China (50576008, 50876016 and 51006015) and China Postdoctoral Science Foundation (20100470070) are gratefully acknowledged. We acknowledge the reviewers' comments and suggestions very much, which are valuable in improving the quality of our manuscript.

\section{CONFLICT OF INTEREST}

Declared none.

\section{REFERENCES}

[1] Choi, S.U.S.; Eastman, J.A. In: Developments and applications of non-newtonian flows; Singer, D.A.; Wang, H.P., Eds.; ASME: New York, USA, 1995; pp. 99-105.

[2] Vergeles, M.; Keblinski, P.; Koplik, J.; Banavar, J.R. Stokes drag at the molecular level. Phys.Rev.Lett., 1995, 75(2), 232-235.

[3] Vergeles, M.; Keblinski, P.; Koplik, J.; Banavar, J.R. Stokes drag and lubrication flows: a molecular dynamics study. Phys.Rev.E., 1996, 53(5), 4852-4864. 
[4] Nagayama, G.; Cheng, P. Effects of interface wettability on microscale flow by molecular dynamics simulation. Int. J. Heat Mass Tran., 2004, 47(3), 501-513.

[5] Xue, L.; Keblinski, P.; Phillpot, S.R.; Choi, S.U.S.; Eastman, J.A. Effects of interface effect of liquid layering at the liquid-solid interface on thermal transport. Int. J. Heat Mass Tran., 2004, 47(19-20), 4277-4284.

[6] Kamali, R.; Kharazmi, A. Molecular dynamics simulation of surface roughness effects on nanoscale flows. Int. J. Therm Sci., 2011, 50(3), 226-232.

[7] Ahadian, S.; Mizuseki, H.; Kawazoe, Y. An efficient tool for modeling and predicting fluid flow in nanochannels. Int. J. Chem. Phys., 2009, 131(18), 184506.
[8] Maroo, S.C.; Chung, J.N. A novel fluid-wall heat transfer model for molecular dynamics simulations. J. Nanopart. Res., 2010, 12(5), 1913-1924.

[9] Plimpton, S. Fast parallel algorithms for short-range molecular dynamics. J.Comput.Phys., 1995, 117(1), 1-19.

[10] Sarkar, S.; Selvam, R.P. Molecular dynamics simulation of effective thermal conductivity and study of enhanced thermal transport mechanism in nanofluids. J. Appl. Phys., 2007, 102, 074302.

[11] Allen, M.P.; Tildesley, D.J. Computer simulation of liquids; Clarendon Press: Oxford, 1987.

[12] Li, L.; Zhang, W.Y.; Ma, H.B.; Yang, M. An investigation of molecular layering at the liquid-solid interface in nanofluids by molecular dynamics simulation. Phys. Lett. A., 2008, 372(25), $4541-4544$.

(C) Cui et al.; Licensee Bentham Open.

This is an open access article licensed under the terms of the Creative Commons Attribution Non-Commercial License (http://creativecommons.org/licenses/by$\mathrm{nc} / 3.0 /$ ) which permits unrestricted, non-commercial use, distribution and reproduction in any medium, provided the work is properly cited. 